\title{
FAUNA DE CHIRONOMIDAE E OUTROS INSETOS AQUÁTICOS DE AÇUDES DO SEMI-ÁRIDO PARAIBANO, BRASIL
}

\author{
Francisco José Pegado Abílio(1), Alaíde A. Fonseca-Gessner(2), \\ Takako Watanabe ${ }^{(1)} \&$ Romualdo Lunguinho Leite $^{(3)}$
}

\begin{abstract}
Chironomidae fauna and anothers aquatic insects of dams from Paraiban semi-arid, Brazil - With the aim of study the Chironomidae fauna and the anothers aquatic insects of São Mamede - SM dam (06 $55^{\prime}$ S e $37^{\circ}$ 06' W) and São Gonçalo - SG dam (06 우 50 S e 38 19' W), from paraiban semi-arid, qualitatives samples were realized in August and Dezember/ 1995 (dry period) and July/96 (after the maximum of rain). The insect fauna was sampled randomly, next to the aquatic vegetation, with one hand net of $0,50 \mathrm{~mm}$ mesh size. Overall 15 taxa were collected, all of Chironomus gr. decorus was the dominat species of Chironomidae and frenquent in the two dams, which contributed with one maximum abundance of $81.82 \%$ in SG (Dezember/1995). In July/1996 we have also observed the occurrence of Goeldichironomus gr. pictus (1.45\% in SG), Aedokritus sp. (44.20\% in $\mathrm{SG}$ and $0.89 \%$ in SM), Fissimentum sp. (5.07\% in SG) and Asheum sp.

(1) Departamento de Metodologia da Educação, Centro de Educação e Departamento de Sistemática e Ecologia, Centro de Ciências Exatas e da Natureza, Universidade Federal da Paraíba, Rua Maria Rosa Padilha 84, Edifício Aeroville, Ap. 210, Bairro Bessa, CEP: 58037-260 João Pessoa, Paraíba. E-mail: chicopegado@hotmail.com; (2) Departamento de Hidrobiologia, Universidade Federal de São Carlos, Via Washington Luis, Km 235, Caixa Postal 676, CEP: 13565-905 São Carlos, São Paulo; (3) Faculdade de Filosofia Dom Aureliano Matos, Universidade Estadual do Ceará, Rua Jaime Leonel Chaves 688, CEP: 63930-000 Centro, Limoeiro do Norte, Ceará.
\end{abstract}

Com apoio financeiro do CNPq e CAPES. 
(45.33\% in SM). One major abundance of aquatic insects in July/96 it may be related with the lowest values of electric conductivity, alkalinity and hardness, and one highest water level in dams. Then in August and Dezember/95, mainly in SM, was registered high values of electric conductivity, alkalinity, hardness and sulphate, and the lowest abundance of aquatic insects. However, due to environmental factors result in effects at organism, population and community level.

Key words: Aquatic insects, Chironomidae, Chironomusgr. decorus, semi-arid

\section{Resumo}

Com o objetivo de estudar a fauna de Chironomidae e outros insetos aquáticos dos açudes São Mamede - SM (06ํ5' S e 37ํ06' W) e São

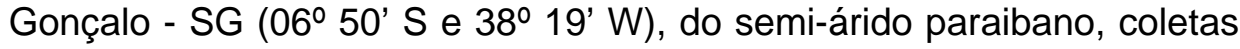
qualitativas foram realizadas em agosto e dezembro/95 (período seco) e julho/96 (após o pico máximo de chuvas da região). Foram feitos arrastos junto a vegetação da margem, utilizou-se para isso um pegador manual com 0,50 mm de abertura de malha. Registrou-se a participação de 15 taxa, dos quais Chironomus gr. decorus foi à espécie de Chironomidae dominante e freqüente nos dois açudes, contribuindo com uma abundância máxima de 81,82\% em SG (dezembro/1995). Já no mês de julho/1996, constatou-se também a ocorrência de Goeldichironomus gr. pictus (1,45\% em SG), Aedokritus sp. (44,20\% em SG e 0,89\% em SM), Fissimentum sp. (5,07\% em SG) e Asheum sp. (45,33\% em SM). Uma maior abundância de insetos aquáticos em julho/96 pode estar relacionada com os valores mais baixos de condutividade elétrica da água, alcalinidade e dureza, e um elevado nível d'água dos açudes. Já nos meses de agosto e dezembro/ 95, principalmente em SM, constatou-se elevados valores de condutividade, alcalinidade, dureza e sulfato, e uma baixa abundância de insetos aquáticos. A acentuada escassez populacional e taxonômica nesses açudes são fortemente influenciadas pelas condições ambientais.

Palavras-chave: Insetos aquáticos, Chironomidae, Chironomus gr. decorus, semi-arido 


\section{Introdução}

A presença e a abundância de várias espécies em uma determinada comunidade e suas interações proporcionam indícios sobre o funcionamento do ecossistema (Wetzel, 1975).

Padrões de colonização e sucessão em hábitats aquáticos tem sido investigados para alguns grupos taxonômicos incluindo Coleoptera, Hemiptera, Odonata e Diptera. A abundância e a distribuição de Chironomidae (Diptera) são fortemente influenciadas pelas condições físicas, químicas e tróficas do hábitat, e por isso são particulamente usados para estudar os efeitos das dinâmicas espacial e temporal. Os Chironomidae são muitas vezes os primeiros colonizadores de novos hábitats, sendo que a sua composição muda com as condições do habitat e ao longo do tempo (Botts, 1997).

As larvas de Chironomidae podem ocupar posição importante na dinâmica trófica de ecossistemas aquáticos de água doce, devido a sua abundância númerica e o papel na reciclagem de nutrientes nos sedimentos. Elas alteram a composição da fina matéria orgânica particulada e provêm importantes subsídios energéticos para os predadores (Sankarperumal \& Pandian, 1992). Além disso, como eles têm um ciclo de vida suficientemente longo e movem-se pouco, eles integram muito bem vários processos biológicos aquáticos e podem servir como indicadores de condições ambientais (Seminara \& Bazzanti, 1988).

Vários autores têm classificados os lagos naturais com base na fauna de Chironomidae (Peterson \& Fernando, 1969), além disso, essa comunidade de insetos aquáticos é utilizada como avaliadoras do estado de eutrofização do corpo aquático (Warwick, 1980; Zinchenko, 1992). O alto número de espécies de dipteros, junto com sua extraordinária diversidade de requerimentos ecológicos, faz destes um dos principais grupos de interesse para usar como indicadores ecológicos (Gallardo \& Prenda, 1994)

A ausência de conhecimento a respeito das comunidades bentônicas dos açudes do semi-árido paraibano e particularmente dos Chironomidae, frequentemente os mais abundantes e amplamente distribuídos nos diversos habitats fluviais (Coffman, 1984; Crauston, 1995), direcionou este trabalho, que teve como objetivo estudar a composição da fauna de Chironomidae e outros insetos aquáticos dos açudes São Mamede (São Mamede) e São Gonçalo (Sousa) e o possível efeito das variáveis físicas e químicas sobre essa comunidade. 


\section{Material e Métodos}

\section{Características das áreas de estudo:}

Açude São Mamede: Localizado no município de São Mamede (06ํ5' S e 37을 'W; Altitude $290 \mathrm{~m}$ ), na região fisiográfica do Seridó paraibano (Caatinga), o açude concluído em 1969, tem uma capacidade de acumulação de $15.791 .280 \mathrm{~m}^{3}$. O clima da região segundo a classificação de Koeppen é do tipo Bsh (semi-árido quente com chuvas de verão), apresentando de 9 a 11 meses secos e $300 \mathrm{~mm}$ de precipitação/ano. Este açude faz parte da Bacia Hidrográfica do Rio Sabugi $\left(192,80 \mathrm{~km}^{2}\right)$ e o tipo de solo é classificado como Brunos não cálcicos (Atlas Geográfico do Estado da Paraíba, 1985; Relatório do DNOCS, 1996).

Açude São Gonçalo: o açude localizado no município de Sousa ( $06^{\circ} 50^{\prime}$ S e 38우 19' W; Altitude $235 \mathrm{~m}$ ), foi construído em 1936, tendo uma capacidade de acumulação de $44.600 .000 \mathrm{~m}^{3}$. É um reservatório de multi-uso, sendo aproveitado para abastecimento urbano, irrigação e lazer. O município está localizado na região fisiográfica do sertão de Piranhas, Alto Piranhas (Caatinga) e o clima da região segundo a classificação de Koeppen é do tipo Aw' - quente e úmido com chuvas de verão/outono, com período seco que varia de 7 a 8 meses ( 600 a $800 \mathrm{~mm}$ de precipitação/ano). Este açude faz parte da Bacia Hidrográfica do Rio Piranhas $\left(171 \mathrm{~km}^{2}\right)$ e o tipo de solo é classificado como Brunos não cálcicos (Atlas Geográfico do Estado da Paraíba, 1985; Relatório do DNOCS, 1996).

As coletas dos dados foram realizadas nos meses de agosto e dezembro/ 1995 e julho/1996, os dois primeiros no período de seca e o último após o período chuvoso.

Variáveis Físicas e Químicas da água: Temperatura da Água $\left({ }^{\circ} \mathrm{C}\right)$ determinada através de um termômetro de mercúrio com $0,5^{\circ} \mathrm{C}$ de precisão; $\mathrm{pH}$ - utilizou-se um medidor de pH marca Horiba B-213, digital portátil; Oxigênio Dissolvido $\left(\mathrm{mg} \mathrm{O}_{2} / \mathrm{l}\right)$ determinado através do método clássico de Winkler e a Alcalinidade $\left(\mathrm{mgCaCO}_{3} /\right)$ determinada por titulação, ambos através de métodos descritos em Golterman et al., (1978); Condutividade Elétrica $(\mu \mathrm{S} / \mathrm{cm})$ - utilizou-se um conditivímetro Analyser; Dureza Total $\left(\mathrm{mgCaCO}_{3} / l\right)$ - determinada por titulação através do método descrito em Standard Methods (APHA, 1995); O Amônio e o Nitrito foram determinados segundo Mackereth et al. (1978); Nitrato - 
determinado segundo as técnicas descritas em Rodier (1975). Os dados pluviométricos foram obtidos no LMRS-PB (Laboratório de Meteorologia, Recursos Hídricos e Sensoriamento Remoto da Paraíba - Campina Grande).

Fauna de Macroinvertebrados: Foram feitas amostragens qualitativas do zoobentos, utilizando-se um pegador manual com uma rede de malha de $0,50 \mathrm{~mm}$. O sedimento coletado, em três pontos previamente fixados, foi lavado em peneiras de $1 \mathrm{~mm}$ e $0,20 \mathrm{~mm}$. As amostras foram colocadas em potes plásticos e o material fixado em formol a $10 \%$. No laboratório foi feito a triagem do material em estereomicroscópio Zeiss. Os organismos preservados em álcool a $70 \%$ foram indentificados seguindo: Merrit \& Cummins (1984) e Trivinho-Strixino \& Strixino (1995).

\section{Resultados e Discussões}

Durante o período de estudo foram registrados 15 taxa, com 5 gêneros para Chironomidae (Tab. I).

Chironomus gr. decorus foi a espécie de Chironomidae dominante e freqüente nos dois açudes, contribuindo com uma abundância máxima de $81,82 \%$ em SG (dezembro/95). Já no mês de julho/96, além desta observou-se a ocorrência de Goeldichironomus gr. pictus (1,45\% em SG), Aedokritus sp. (44,20\% em SG e 0,89\% em SM), Fissimentum sp. $(5,07 \%$ em SG) e Asheum sp. (45,33\% em SM).

Além dos Chironomidade, registrou-se a ocorrência de outras duas famílias de Diptera (Stratiomyidae e Ceratopogonidae). Entre os Odonata Gomphidae, Coenagrionidae e Aeshnidae, os Gomphidae contribuiram com um máximo de 52,17 \% em SM (dezembro/95). Em agosto/95 em SG, apenas larvas e adultos de Hydrophilidae (Coleoptera) foram registrados. Já em julho/96 esta família contribuiu com 17,35 \% para o total da fauna de insetos aquáticos. Além de Hydrophilidae, observou-se a ocorrência de Noteridae neste açude. Os Ephemeroptera (Caenidae) apenas foram registrados em SM, contribuindo com máximo de 8,69 \% (dezembro/95). Os Hemiptera Ranatra sp. (Nepidae, apresentou uma abundância máxima de 18,18 \%) e Belostoma sp. (Belostomatidae) apenas foram encontrados em SG. 
Tabela I

Abundância relativa (\%) da fauna de insetos aquáticos de açudes do semi-árido paraibano

\begin{tabular}{|c|c|c|c|c|c|c|}
\hline \multirow[t]{2}{*}{ TAXA } & \multicolumn{2}{|c|}{ Agosto/1995 } & \multicolumn{2}{|c|}{ Dezembro/1995 } & \multicolumn{2}{|c|}{ Julho/1996 } \\
\hline & SM & SG & SM & SG & SM & SG \\
\hline \multicolumn{7}{|l|}{ DIPTERA } \\
\hline \multicolumn{7}{|l|}{ Chironomidae } \\
\hline Chironomus gr. decorus & 69,69 & & 39,14 & 81,82 & 51,11 & 21,74 \\
\hline Goeldichironomus gr. pictus & & & & & & 1,45 \\
\hline Aedokritus sp. & & & & & 0,89 & 44,20 \\
\hline Fissimentum sp. & & & & & & 5,07 \\
\hline Asheum sp. & & & & & 45,33 & \\
\hline Stratiomyidae & & & & & & 2,90 \\
\hline Ceratopogonidae & & & & & 0,89 & \\
\hline \multicolumn{7}{|l|}{ ODONATA } \\
\hline Gomphidae & 24,25 & & 52,17 & & & \\
\hline Coenagrionidae & & & & & & 1,45 \\
\hline Aeshnidae & & & & & & 1,45 \\
\hline \multicolumn{7}{|l|}{ EPHEMEROPTERA } \\
\hline Caenidae & 6,06 & & 8,69 & & & \\
\hline \multicolumn{7}{|l|}{ COLEOPTERA } \\
\hline Hydrophilidae & & 100 & & & 1,78 & 17,39 \\
\hline Noteridae & & & & & & 2,90 \\
\hline \multicolumn{7}{|l|}{ HEMIPTERA } \\
\hline Nepidae & & & & & & \\
\hline Ranatra sp. & & & & 18,18 & & \\
\hline Belostomatidae & & & & & & \\
\hline Belostoma sp. & & & & & & 1,45 \\
\hline
\end{tabular}

SG: São Gonçalo; SM: São Mamede. 
Na tabela II estão representados os valores das variáveis físicas e químicas da água dos açudes São Mamede e São Gonçalo nos meses de agosto e dezembro/95 e julho/96.

A ausência de amônio, não determinado pelo método, nas primeiras amostragens, provavelmente esteve relacionada com o consumo pelo fitoplâncton e pelas macrófitas aquáticas, uma vez que os teores de oxigênio dissolvido mantiveram-se relativamente altos.

As flutuações dos valores de condutividade elétrica, alcalinidade e dureza da água, dentro dos ambientes estão associadas com o regime das chuvas da região (Fig. 1). O aumento dos valores, durante o período de estiagem é provocado pela alta taxa de evaporação da água, que contribui para um aumento da precipitação de sais, enquanto que aumento da precipitação pluviométrica tende a diminuir os valores dessas variáveis pelo efeito da diluição.

De acordo com Moredjo (1998) as atividades de irrigação nas margens do açude São Gonçalo, a temperatura e as características geoquímicas do solo, contribuem para o aumento da condutividade elétrica neste ambiente. Além disso, é comum nesta localidade a utilização de produtos químicos (NPK, Superfosfato e Uréia), e a irrigação das lavouras tem contribuído para uma maior entrada de substâncias químicas no açude, o que pode influenciar a fauna bentônica.

Altas concentrações de sulfato e/ou amônia podem ser tóxicas para os insetos aquáticos e outros invertebrados bentônicos (Popp \& Hoagland, 1995). Assim como as concentrações de Nitrato e Nitrito pode limitar a diversidade de dipteros quironomídeos. Portanto, várias espécies são consideradas como indicadoras ecológicas do estoque de nitrogênio dissolvido e/ou de altos valores de salinidade (Gallardo \& Prenda, 1994).

Uma maior abundância de insetos aquáticos em julho/96 pode estar relacionada com os valores mais baixos de condutividade elétrica da água (193 mS/cm para SG e $716 \mathrm{mS} / \mathrm{cm}$ para SM), alcalinidade (12 $\mathrm{mg} \mathrm{CaCO}_{3} /$ I e $13 \mathrm{mg} \mathrm{CaCO} /$ /, respectivamente para SG e SM) e dureza (59 $\mathrm{mg} \mathrm{CaCO}_{3}$ / I para SG), um elevado nível d'agua dos açudes e uma grande quantidade de macrófitas aquáticas (Salvinia auriculata e Pistia stratiotes). Já nos meses de agosto e dezembro/95 (período seco), principalmente em SM, os valores de condutividade foram elevados $(2130 \mu \mathrm{S} / \mathrm{cm})$, alcalinidade $e$ dureza $\left(315 \mathrm{CaCO}_{3} / \mathrm{l}\right)$, para ambas as variáveis ) e sulfato $(17,09 \mathrm{mg} / \mathrm{l})$, o que correspondeu a uma baixa abundância de insetos aquáticos. Neste período os elevados valores, dessas variáveis físicas e químicas da água, podem ter afetado a colonização e a abundância de insetos aquáticos. 


\section{Tabela II}

Variáveis físicas e químicas da água (média de 3 pontos de coleta) de açudes do semi-árido paraibano

\begin{tabular}{l|c|c|c|c|c|c}
\hline Variáveis Físicas e químicas & \multicolumn{2}{|c|}{ Agosto/95 } & Dezembro/95 & \multicolumn{2}{c}{ Julho/96 } \\
\cline { 2 - 7 } & SG & SM & SG & SM & SG & SM \\
\cline { 2 - 7 } Temperatura $\left({ }^{\circ} \mathrm{C}\right)$ & 26.8 & 28 & 29.7 & 32 & 26.3 & 28 \\
Oxigênio Dissolvido $\left(\mathrm{mg} \mathrm{I}^{-1}\right)$ & 6.20 & 8.97 & 5.92 & 6.36 & 3.99 & 6.60 \\
Condutividade Elétrica $\left(\mu \mathrm{S} \mathrm{cm}^{-1}\right)$ & 218 & 1358 & 260 & 2130 & 193 & 716 \\
$\mathrm{pH}$ & 7.13 & 7.23 & 7.82 & 8.39 & 7.57 & 7.59 \\
Alcalinidade $\left(\mathrm{mg} \mathrm{CaCO}_{3} \mathrm{I}^{-1}\right)$ & 71 & 141 & 75 & 315 & 12 & 13 \\
Dureza Total $\left(\mathrm{mg} \mathrm{CaCO}_{3} \mathrm{I}^{-1}\right)$ & 72 & 118 & 84 & 315 & 59 & 118 \\
Amônio $\left(\mu \mathrm{gl}^{-1}\right)$ & 0.00 & 0.00 & 0.00 & 0.00 & 26.77 & 34.55 \\
Nitrito $\left(\mu \mathrm{I} \mathrm{I}^{-1}\right)$ & 1.12 & 3.71 & 4.15 & 3.28 & 6.16 & 6.64 \\
Nitrato $\left(\mu \mathrm{g} \mathrm{I}^{-1}\right)$ & 271.09 & 197.20 & 83.53 & 174.47 & 122.67 & 146 \\
Sulfato $\left(\mathrm{mg} \mathrm{I}^{-1}\right)$ & 0.62 & 17.09 & 0.62 & 13.72 & 1.30 & 4.38 \\
Orto-Fostato $\left(\mu \mathrm{g} \mathrm{I}^{-1}\right)$ & 2.57 & 9.71 & 14.00 & 6.85 & 2.57 & 10.66 \\
\hline
\end{tabular}

SG: São Gonçalo; SM: São Mamede.

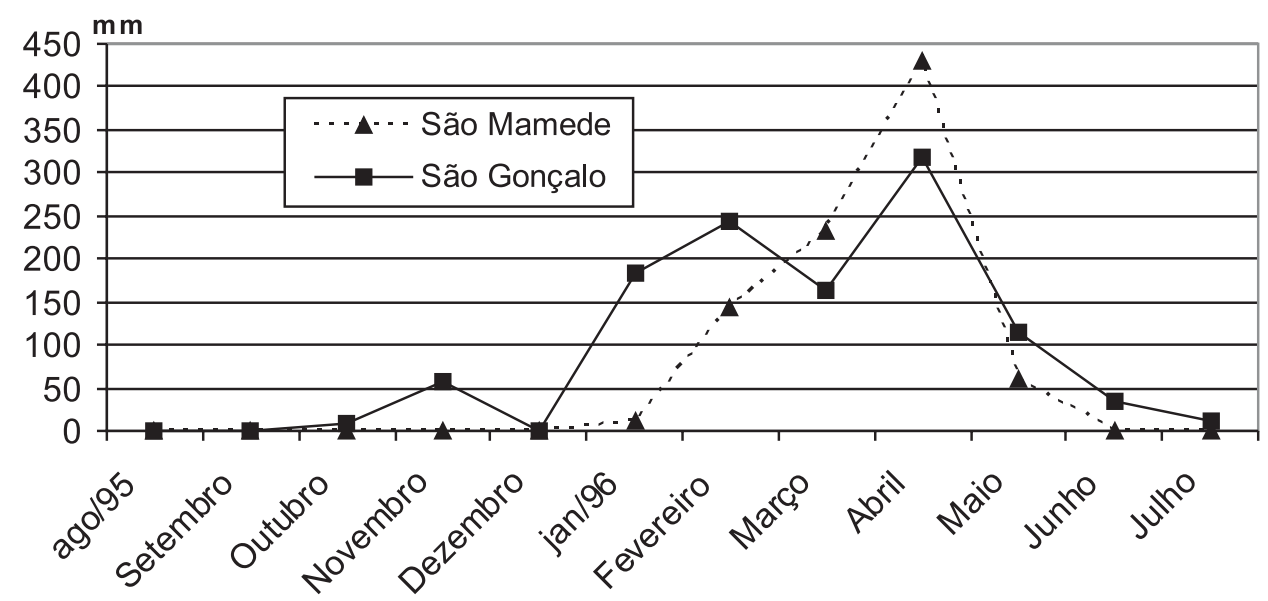

Figura 1: Índices pluviométricos dos municípios de São Mamede e Sousa (São Gonçalo) no período de agosto de 1995 a julho de 1996. 


\section{Referências Bibliográficas}

APHA, 1995. Standard Methods for examination of water and wastewater. 19ํe․ Washington, American Public, Health Association. $1193 \mathrm{p}$.

Atlas Geográfico do Estado da Paraíba, 1985. João Pessoa: Secretaria da Educação, 100 p.

Botts, P.S., 1997. Spatial pattern, patch dynamics and successional change: chironomid assemblages in a Lake Erie coastal wetland. Freshwater Biol. 37:277-286.

Coffman, W.P., 1984. Chironomidae. p.345-376, In: An introduction to the aquatic insects of North America. Merrit \& Cummins (ed). Kendall/ Hunt Publ. Co. Dubuque, lowa. 722 p.

Cranston, P.S., 1995. Introductio p.1-7. In: The Chironomidae: the biology and ecology of non-biting midges. Amitage, Cranston \& Pinder (ed). Chapman \& Hall, New York. 572 p.

Gallardo, A. \& Prenda, J., 1994. Influence of some environmental factors on the freshwater macroinvertebrates distribution in two adjacent river basins under Mediterranean climate. I. dipteran larvae (excepting chironomids and simuliids) as ecological indicators. Arch. Hydrobiol. 131:435-447.

Golterman, H.L.; Clymo. R.S. \& Ohnstad, M.A.M., 1978. Methods for physical and chemical analysis of fresh water. 2nd ed. Oxford. Blackwell Sci. Publ. 214 p.

Mackereth, F.J.H.; Heron, J. \& Talling, J.F., 1978. Water analysis: some revised methods for limnologists. Dorset, Freshwater Biol. Ass. 121 p.

Merrit, R.W. \& Cummins, K.W., 1984. An introduction to the aquatic insects of North America. Kendall/Hunt Publ. Co., Dubuque, lowa. 722 p.

Moredjo, A., 1998. Avaliação dos efeitos das atividades humanas sobre o estado trófico dos açudes paraibanos, com ênfase na utilização 
da comunidade zooplanctônica como bioindicador. Dissertação de mestrado, PRODEMA-UFPB, João Pessoa-PB. 136 p.

Peterson, C.G. \& Fernando, C.H., 1969. The macro-invertebrate colonization of a small reservoir in Eastern Canada. Verh. Internat. Verein. Limnol. 17:126-136.

Popp, A. \& Hoagland, K.D., 1995. Changes in benthic community composition in response to reservoir aging. Hydrobiol. 306:159-171.

Relatório do DNOCS., 1996. João Pessoa, Paraíba. 35 p.

Rodier, J., 1975. L'analyse de l'eau: eaux naturelles, euax résiduales, eaux de mer. Volume 1, 5ed. Dunod (Ed.) Paris. 692 p.

Sankarperumal, G. \& Pandian, T.J., 1992. Larval abundance of Chironomus circumdatus in relation to biotic and abiotic factors. Hydrobiol. 246:205-212.

Seminara, M. \& Bazzanti, M., 1988. Trophic level assessment of profundal sediments of the artificial lake Campotosto (Central Italy), using midge larval community (Diptera: Chironomidae). Hydrobiol. Bull. 22 :183-193.

Trivinho-Strixino, S. \& Strixino, G., 1995. Larvas de Chironomidae (Diptera) do Estado de São Paulo: Guia de identificação e diagnose dos gêneros. PPG-ERN/UFSCar, São Carlos, São Paulo. 229 p.

Verdonschot, P.F.M.; Real, M. \& Schot, J.A., 1992. Chironomids and regional water types. Nether. J. Aquat. Ecol. 26:513-520.

Warwick, W.F., 1980. Pasqua Lake, Souteastern Saskatchewan: a preliminary assessment of trophic status and contamination based on the Chironomidae (Diptera). p. 255-267. D.A. Murray Ed., Pergamon press Oxford \& New York.

Wetzel, R.G., 1975. Limnology. Philadelphia. W.B. Saunder Co. 743 p.

Zinchenko, T.D., 1992. Long-term (30 years) dynamics of Chironomidae (Diptera) fauna in the Kuibyshev water resevoir associated with eutrophication processes. Nether. J. Aquat. Ecol. 26:533-542. 